\title{
Rapid Solidification in Thin-Film Al-Cu Alloys: Capturing the Dynamics with Time-Resolved In Situ TEM
}

\author{
Joseph T. McKeown ${ }^{1}$, Kai Zweiacker ${ }^{2}$, Thomas LaGrange ${ }^{1}$, Bryan W. Reed ${ }^{1}$, Can Liu ${ }^{2}$, Jörg M.K. \\ Wiezorek $^{2}$, and Geoffrey H. Campbell ${ }^{1}$ \\ ${ }^{1}$ Condensed Matter and Materials Division, Lawrence Livermore National Laboratory, Livermore, CA, \\ USA \\ ${ }^{2}$ Department of Mechanical Engineering and Materials Science, University of Pittsburgh, Pittsburgh, \\ PA, USA
}

Rapid solidification of metals and alloys has been widely recognized as a viable processing route to obtain unique microstructures with potentially advantageous properties that are not obtainable with conventional solidification processes. Previously, the dynamics of rapid solidification in pure Al [1] and $\mathrm{Al}-\mathrm{Cu}$ alloy [2] thin films were characterized using the dynamic transmission electron microscope (DTEM) in its singleshot, single-image acquisition mode. Recent upgrades to the DTEM instrument enable a single-shot, multiple-image acquisition mode (so-called movie mode) [3], which allows study of microstructural evolution and kinetics in far greater detail. Movie-mode DTEM provides higher data throughput and a reduction in uncertainty and experimental error while following complex, irreversible processes in time.

Here, we present results of in situ DTEM investigations of rapid solidification in thin-film Al-Cu alloys spanning a composition range from Al-3at. $\% \mathrm{Cu}$ to approximately the eutectic composition (Al17 at. $\% \mathrm{Cu}$ ). Figure 1 shows a time-delay sequence of images recorded as an 80 -nm-thick $\mathrm{Al}-3 \mathrm{at} . \% \mathrm{Cu}$ thin film re-solidifies after pulsed-laser melting. The upper and lower rows of images were recorded from two separate solidification experiments performed with initial delay times of $0 \mu \mathrm{s}$ and $20 \mu \mathrm{s}$, respectively. Each experiment spans $20.4 \mu \mathrm{s}$ and produces 9 images using a $2.5-\mu$ s interframe spacing and a 50-ns electron pulse duration. Figure 2 shows a plot of the time evolution of the melt pool area and solidification front velocity, obtained directly from the time-resolved images of Figure 1. The velocity can be related to the microstructure evolution seen in Figure 1. The solidification front accelerates up to $>2 \mathrm{~m} / \mathrm{s}$ as the alloy film solidifies with a columnar structure. Solidification completes by $\sim 31 \mu$ s after pulsed-laser melting. The details of microstructural evolution and solidification kinetics for all alloy compositions investigated will be analyzed and discussed, along with post-mortem ex-situ characterization of the resultant microstructure.

\section{References}

[1] A.K. Kulovits et al., Philosophical Magazine Letters 91 (2011) 287.

[2] J.T. McKeown et al., Acta Materialia 65 (2014) 56.

[3] T. LaGrange et al., Micron 43 (2012) 1108.

[4] This work was performed under the auspices of the U.S. Department of Energy by Lawrence Livermore National Laboratory (LLNL) under Contract No. DE-AC52-07NA27344. Activities and personnel at LLNL were supported by the Office of Science, Office of Basic Energy Sciences, Division of Materials Sciences and Engineering of the U.S. Department of Energy. Activities and personnel at the University of Pittsburgh were supported by the National Science Foundation, Division of Materials Research, Metals \& Metallic Nanostructures program through Grant No. DMR 1105757. 


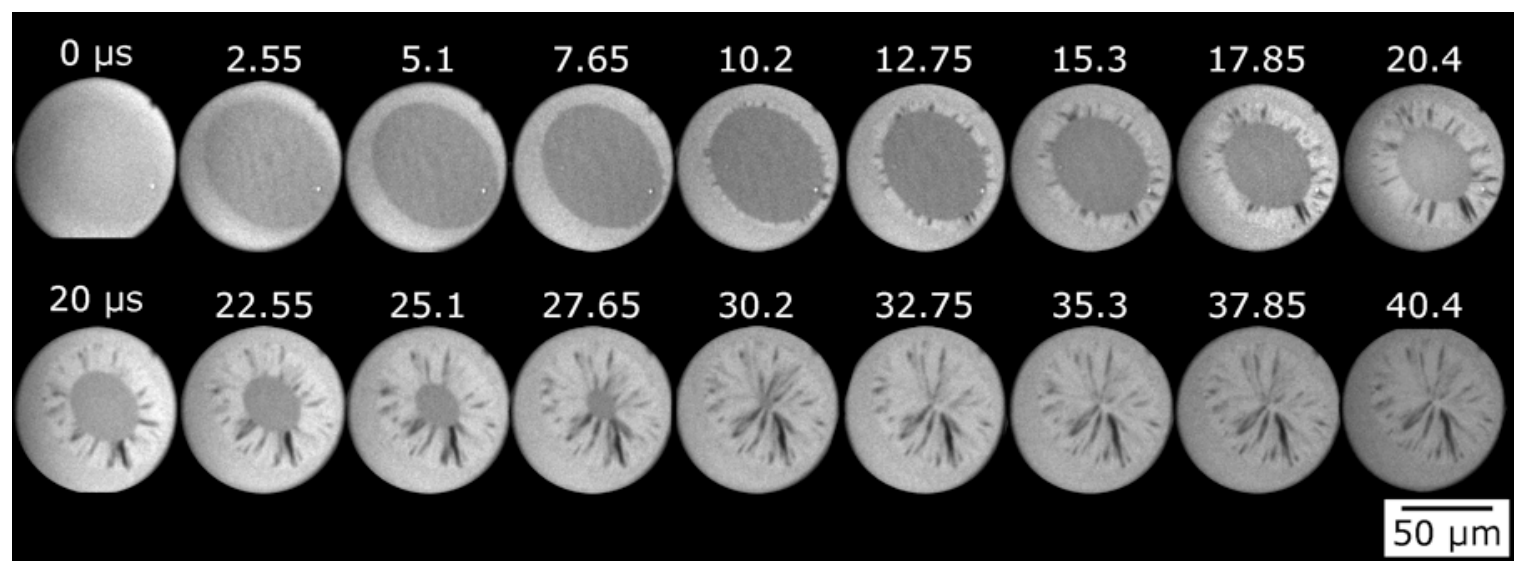

Figure 1. Dynamic time-delay sequence of images recorded as an $80-\mathrm{nm}$-thick $\mathrm{Al}-3 \mathrm{at} . \% \mathrm{Cu}$ thin film resolidifies after pulsed-laser melting. The indicated times above each image are the delays (in $\mu \mathrm{s}$ ) between the peak of the Gaussian laser pulse that melted the film and the 50-ns electron pulse used to form the image. The upper and lower rows of images were recorded from two separate solidification experiments performed with initial delay times of $0 \mu \mathrm{s}$ and $20 \mu \mathrm{s}$, respectively. Each experiment spans $20.4 \mu \mathrm{s}$.

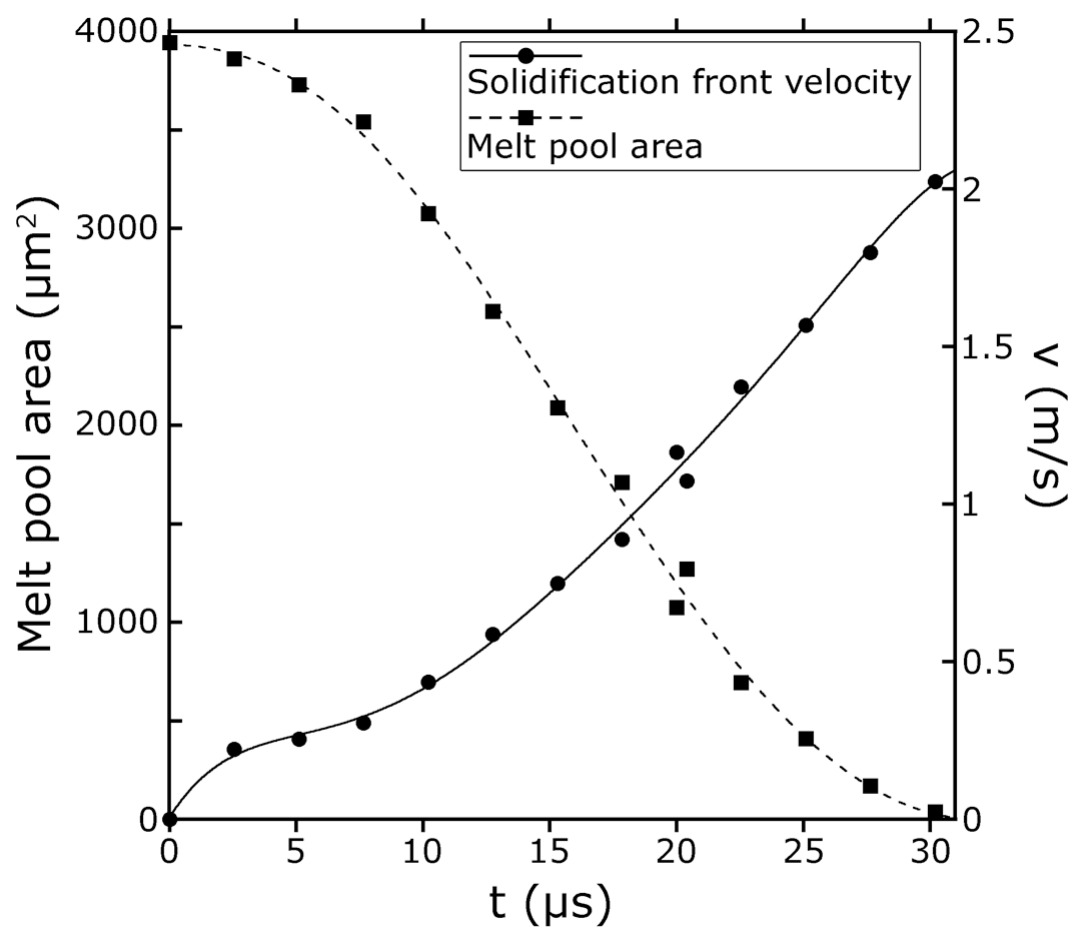

Figure 2. Plot of the melt pool area and solidification front velocity as a function of time, as measured from Figure 1. The plot shows that the solidification front accelerates as the alloy film solidifies. 